\title{
A Hilbert-type integral inequality under configuring free power and its applications
}

\section{Qiong Liu' ${ }^{1 *}$ (D)}

\section{"Correspondence:}

liuqiongxx13@163.com

${ }^{1}$ College of Science, Shao yang

University, Shao yang, P.R. China

\section{Springer}

\begin{abstract}
By using the method of weight function, the technique of real analysis, and the theory of special functions, a multi-parameter Hilbert-type integral inequality and its equivalent form are established, and their constant factors are proved to be the best possible. The expressions of operator with norm are given. As an application, relevant results in the references and some new inequalities are obtained by assigning some parameter values.
\end{abstract}

MSC: 26D15

Keywords: Hilbert-type integral inequality; Weight function; Whittaker function; Operator; Application

\section{Introduction}

If $f, g:(0, \infty) \rightarrow \mathbb{R}$ are non-negative integrable functions, satisfying $0<\int_{0}^{\infty} f^{2}(x) d x<\infty$, $0<\int_{0}^{\infty} g^{2}(y) d y<\infty$, the celebrated Hilbert integral inequality is as follows (see [1]):

$$
\int_{0}^{\infty} \int_{0}^{\infty} \frac{f(x) g(y)}{x+y} d x d y<\pi\left\{\int_{0}^{\infty} f^{2}(x) d x\right\}^{\frac{1}{2}}\left\{\int_{0}^{\infty} g^{2}(y) d y\right\}^{\frac{1}{2}}
$$

where the constant factor $\pi$ is the best possible. Inequality (1) is very important in harmonic analysis and theory of partial differential equations (see $[1,2])$. During decades, inequality (1) has been extensively studied by numerous authors, evolved into a lot of meaningful results, which include the research of parametric quantization, mixed kernels, homogeneous kernels and non-homogeneous kernels, the extensions of fractal space, etc. (see [3-15]). In 2011, Yang gave an integral inequality of Hilbert type with exponential kernel as follows (see [16]):

$$
\int_{0}^{\infty} \int_{0}^{\infty} e^{-x y} f(x) g(y) d x d y<\sqrt{\pi}\left\{\int_{0}^{\infty} f^{2}(x) d x\right\}^{\frac{1}{2}}\left\{\int_{0}^{\infty} g^{2}(y) d y\right\}^{\frac{1}{2}},
$$

where the constant factor $\sqrt{\pi}$ is the best possible.

In this paper, by using the method of weight function, the technique of real analysis, and the theory of special function, a Hilbert-type integral inequality and its equivalent form with the kernel as $\frac{(\min \{1, x y\})^{\alpha}(\max \{1, x y\})^{\beta}}{e^{\gamma x y}}$ are given, and their optimum constant factor in relation to Whittaker function and the application of the obtained results are briefly discussed.

(c) The Author(s) 2019. This article is distributed under the terms of the Creative Commons Attribution 4.0 International License (http://creativecommons.org/licenses/by/4.0/), which permits unrestricted use, distribution, and reproduction in any medium, provided you give appropriate credit to the original author(s) and the source, provide a link to the Creative Commons license, and indicate if changes were made. 
We configured with power parameters for each factor of the integral kernel. Besides, we introduce a free parameter $\theta$ (it can take any real number) when using the weight function method based on "Hardy interpolation problem". In practical applications, the conditions $0<\int_{0}^{\infty} x^{p \theta-1} f^{p}(x) d x<\infty$ and $0<\int_{0}^{\infty} y^{q \theta-1} g^{q}(y) d y<\infty$ in the obtained results are easily met by selecting the parameter $\theta$ as needed. On the other hand, a series of Hilberttype integral inequalities with single kernels, mixed kernels, and compound kernels can be obtained by selecting appropriate parameter $\theta$ and other parameter values, so that the obtained results can be used more widely.

\section{Preliminaries}

Some special functions are required in the following deduction (see [17]).

(1) Suppose that $\operatorname{Re}(s)>0$, then gamma function $\Gamma(s)$ and incomplete gamma function $\Gamma(s, a)(a>0)$ are defined by the expressions

$$
\begin{aligned}
& \Gamma(s):=\int_{0}^{\infty} e^{-t} t^{s-1} d t, \\
& \Gamma(s, a):=\int_{a}^{\infty} e^{-t} t^{s-1} d t .
\end{aligned}
$$

(2) Beta function $B(u, v)(u, v>0)$ is defined by the expression

$$
B(u, v):=\int_{0}^{1}(1-t)^{u-1} t^{\nu-1} d t=\frac{\Gamma(u) \Gamma(v)}{\Gamma(u+v)} .
$$

(3) Confluent hypergeometric function (also called Kummer function) ${ }_{1} F_{1}(\lambda, \mu, z)$ $(\lambda, \mu, z>0)$ is defined by the expression

$$
{ }_{1} F_{1}(\lambda, \mu, z):=\sum_{n=0}^{\infty} \frac{(\lambda)_{n}}{n !(\mu)_{n}} z^{n}=\frac{\Gamma(\mu)}{\Gamma(\lambda)} \sum_{n=0}^{\infty} \frac{z^{n} \Gamma(n+\lambda)}{n ! \Gamma(n+\mu)},
$$

here, the mark $(x)_{n}=x(x+1) \cdots(x+n-1)=\frac{\Gamma(n+x)}{\Gamma(x)}(x>0)$. If $\gamma>0, \alpha>\theta-1$, by (6), we obtain

$$
{ }_{1} F_{1}(1,2+\alpha-\theta, \gamma)=\Gamma(2+\alpha-\theta) \sum_{n=0}^{\infty} \frac{\gamma^{n}}{\Gamma(n+2+\alpha-\theta)} .
$$

(4) Whittaker function $M(k, m, z)$ is defined as

$$
M(k, m, z):=z^{m+\frac{1}{2}} e^{-\frac{z}{2}}{ }_{1} F_{1}\left(m-k+\frac{1}{2}, 2 m+1, z\right) .
$$

By (7) and (8), we have

$$
M\left(\frac{\alpha-\theta}{2}, \frac{\alpha-\theta}{2}+\frac{1}{2}, \gamma\right)=\gamma^{\frac{\alpha-\theta}{2}+1} e^{-\frac{\gamma}{2}} \Gamma(2+\alpha-\theta) \sum_{n=0}^{\infty} \frac{\gamma^{n}}{\Gamma(n+2+\alpha-\theta)} .
$$

When $\gamma>0, \alpha>\theta-1$, by (5) and (9), we find

$$
I_{1}=\int_{0}^{1} e^{-\gamma t} t^{\alpha-\theta} d t
$$




$$
\begin{aligned}
& =e^{-\gamma} \int_{0}^{1} e^{\gamma(1-t)} t^{\alpha-\theta} d t \\
& =e^{-\gamma} \sum_{n=0}^{\infty} \frac{\gamma^{n}}{n !} \int_{0}^{1}(1-t)^{n} t^{\alpha-\theta} d t \\
& =e^{-\gamma} \sum_{n=0}^{\infty} \frac{\gamma^{n}}{n !} \frac{\Gamma(n+1) \Gamma(1+\alpha-\theta)}{\Gamma(n+2+\alpha-\theta)} \\
& =\frac{\gamma^{\frac{\theta-\alpha}{2}-1} e^{-\frac{\gamma}{2}} \Gamma(1+\alpha-\theta)}{\Gamma(2+\alpha-\theta)}\left[\gamma^{\frac{\alpha-\theta}{2}+1} e^{-\frac{\gamma}{2}} \Gamma(2+\alpha-\theta) \sum_{n=0}^{\infty} \frac{\gamma^{n}}{\Gamma(n+2+\alpha-\theta)}\right] \\
& =\frac{\gamma^{\frac{\theta-\alpha}{2}-1} e^{-\frac{\gamma}{2}}}{1+\alpha-\theta} M\left(\frac{\alpha-\theta}{2}, \frac{\alpha-\theta}{2}+\frac{1}{2}, \gamma\right) .
\end{aligned}
$$

Furthermore, setting $\gamma t=u$, when $\gamma>0, \beta>\theta-1$, by (4), we find

$$
I_{2}=\int_{1}^{\infty} e^{-\gamma t} t^{\beta-\theta} d t=\gamma^{\theta-\beta-1} \int_{\gamma}^{\infty} e^{-u} u^{\beta-\theta} d u=\gamma^{\theta-\beta-1} \Gamma(1+\beta-\theta, \gamma)
$$

Lemma 1 If $p>1, \frac{1}{p}+\frac{1}{q}=1, \theta \in \mathbb{R}, \gamma \geq 0$, when $\gamma>0, \alpha>\theta-1, \theta$ is an arbitrary real number. The weight functions are defined by the following expressions:

$$
\begin{aligned}
& \omega(\alpha, \beta, \gamma, \theta, x):=\int_{0}^{\infty} \frac{(\min \{1, x y\})^{\alpha}(\max \{1, x y\})^{\beta}}{e^{\gamma x y}} \frac{y^{-\theta}}{x^{\theta-1}} d y, \quad x \in(0,+\infty), \\
& \omega(\alpha, \beta, \gamma, \theta, y):=\int_{0}^{\infty} \frac{(\min \{1, x y\})^{\alpha}(\max \{1, x y\})^{\beta}}{e^{\gamma x y}} \frac{x^{-\theta}}{y^{\theta-1}} d x, \quad y \in(0,+\infty),
\end{aligned}
$$

then we have

$$
\begin{aligned}
\omega(\alpha, \beta, \gamma, \theta, x) & \\
& =\omega(\alpha, \beta, \gamma, \theta, y)=C(\alpha, \beta, \gamma, \theta) \\
& = \begin{cases}\frac{1}{\alpha-\theta+1}+\frac{1}{\beta-\theta+1}, & \gamma=0, \alpha>\theta, \beta<\theta-1, \\
\frac{\gamma^{\frac{\theta-\alpha}{2}-1} e^{-\frac{\gamma}{2}}}{1+\alpha-\theta} M\left(\frac{\alpha-\theta}{2}, \frac{\alpha-\theta}{2}+\frac{1}{2}, \gamma\right)+\gamma^{\theta-\beta-1} \Gamma(1+\beta-\theta, \gamma), & \gamma>0, \alpha, \beta>\theta-1 .\end{cases}
\end{aligned}
$$

Proof Setting $x y=t$, when $\gamma=0, \alpha>\theta, \beta<\theta-1$, we have

$$
\begin{aligned}
\omega(\alpha, \beta, \gamma, \theta, x) & :=\int_{0}^{\infty} \frac{(\min \{1, x y\})^{\alpha}(\max \{1, x y\})^{\beta}}{e^{\gamma x y}} \frac{y^{-\theta}}{x^{\theta-1}} d y \\
& =\int_{0}^{1} t^{\alpha-\theta} d t+\int_{1}^{\infty} t^{\beta-\theta} d t \\
& =\frac{1}{\alpha-\theta+1}+\frac{1}{\beta-\theta+1} .
\end{aligned}
$$

When $\gamma>0, \alpha, \beta>\theta-1$, we have

$$
\omega(\alpha, \beta, \gamma, \theta, x):=\int_{0}^{\infty} \frac{(\min \{1, x y\})^{\alpha}(\max \{1, x y\})^{\beta}}{e^{\gamma x y}} \frac{y^{-\theta}}{x^{\theta-1}} d y
$$




$$
\begin{aligned}
& =\int_{0}^{\infty} \frac{(\min \{1, t\})^{\alpha}(\max \{1, t\})^{\beta}}{e^{\gamma t}} t^{-\theta} d t \\
& =\int_{0}^{1} e^{-\gamma t} t^{\alpha-\theta} d t+\int_{1}^{\infty} e^{-\gamma t} t^{\beta-\theta} d t=I_{1}+I_{2} \\
& =\frac{\gamma^{\frac{\theta-\alpha}{2}-1} e^{-\frac{\gamma}{2}}}{1+\alpha-\theta} M\left(\frac{\alpha-\theta}{2}, \frac{\alpha-\theta}{2}+\frac{1}{2}, \gamma\right)+\gamma^{\theta-\beta-1} \Gamma(1+\beta-\theta, \gamma) .
\end{aligned}
$$

Similarly, we can get $\omega(\alpha, \beta, \gamma, \theta, y)=C(\alpha, \beta, \gamma, \theta)$.

Lemma 2 If $p>1, \frac{1}{p}+\frac{1}{q}=1, \theta \in \mathbb{R}, \gamma \geq 0$, when $\gamma=0, \alpha>\theta, \beta<\theta-1$, and when $\gamma>0$, $\alpha, \beta>\theta-1, \varepsilon$ is a sufficiently small positive number, both real functions $\tilde{f}(x), \tilde{g}(y)$ are defined as

$$
\tilde{f}(x)=\left\{\begin{array}{ll}
0, & x \in(0,1), \\
x^{-\theta-\frac{\varepsilon}{p},} & x \in[1, \infty),
\end{array} \quad \tilde{g}(y)= \begin{cases}0, & x \in(1, \infty), \\
y^{-\theta+\frac{\varepsilon}{q}}, & y \in(0,1]\end{cases}\right.
$$

then we have

$$
\begin{aligned}
\tilde{J} \cdot \varepsilon & =\left[\int_{0}^{\infty} x^{p \theta-1} \tilde{f}^{p}(x) d x\right]^{\frac{1}{p}}\left[\int_{0}^{\infty} y^{q \theta-1} \tilde{g}^{p}(y) d y\right]^{\frac{1}{q}} \cdot \varepsilon=1 \\
\tilde{h} \cdot \varepsilon & =\varepsilon \int_{0}^{\infty} \int_{0}^{\infty} \frac{(\min \{1, x y\})^{\alpha}(\max \{1, x y\})^{\beta} \tilde{f}(x) \tilde{g}(y)}{e^{\gamma x y}} d x d y \\
& >C(\alpha, \beta, \gamma, \theta)(1-o(1)) \quad\left(\varepsilon \rightarrow 0^{+}\right) .
\end{aligned}
$$

Proof With the defined functions above, we can easily get

$$
\begin{aligned}
\tilde{J} \cdot \varepsilon & =\left[\int_{0}^{\infty} x^{p \theta-1} \tilde{f}^{p}(x) d x\right]^{\frac{1}{p}}\left[\int_{0}^{\infty} y^{q \theta-1} \tilde{g}^{p}(y) d y\right]^{\frac{1}{q}} \cdot \varepsilon \\
& =\left[\int_{1}^{\infty} x^{-1-\varepsilon} d x\right]^{\frac{1}{p}}\left[\int_{0}^{1} y^{-1+\varepsilon} d y\right]^{\frac{1}{q}} \cdot \varepsilon=1 .
\end{aligned}
$$

Setting $x y=t$, when $\gamma=0$, notice the condition as $\alpha>\theta, \beta<\theta-1$. By Fubini's theorem of commutative integral order (see [18]), we obtain

$$
\begin{aligned}
\tilde{h} \cdot \varepsilon & =\varepsilon \int_{0}^{\infty} \int_{0}^{\infty}(\min \{1, x y\})^{\alpha}(\max \{1, x y\})^{\beta} \tilde{f}(x) \tilde{g}(y) d x d y \\
& =\varepsilon \int_{1}^{\infty} x^{-1-\varepsilon} d x \int_{0}^{x}(\min \{1, t\})^{\alpha}(\max \{1, t\})^{\beta} t^{-\theta+\frac{\varepsilon}{q}} d t \\
& =\varepsilon \int_{1}^{\infty} x^{-1-\varepsilon} d x\left[\int_{0}^{1} t^{\alpha-\theta+\frac{\varepsilon}{q}} d t+\int_{1}^{x} t^{\beta-\theta+\frac{\varepsilon}{q}} d t\right] \\
& =\varepsilon \int_{1}^{\infty} x^{-1-\varepsilon} d x\left[\int_{0}^{1} t^{\alpha-\theta+\frac{\varepsilon}{q}} d t+\int_{1}^{\infty} t^{\beta-\theta+\frac{\varepsilon}{q}} d t-\int_{x}^{\infty} t^{\beta-\theta+\frac{\varepsilon}{q}} d t\right] \\
& =\frac{1}{\alpha-\theta+1+\frac{\varepsilon}{q}}+\frac{1}{\beta-\theta+1+\frac{\varepsilon}{q}}-\varepsilon \int_{1}^{\infty} x^{-1-\varepsilon} d x \int_{x}^{\infty} t^{\beta-\theta+\frac{\varepsilon}{q}} d t \\
& >\frac{1}{\alpha-\theta+1+\frac{\varepsilon}{q}}+\frac{1}{\beta-\theta+1+\frac{\varepsilon}{q}}-\varepsilon \int_{1}^{\infty} x^{-1} d x \int_{x}^{\infty} t^{\beta-\theta+\frac{\varepsilon}{q}} d t
\end{aligned}
$$




$$
\begin{aligned}
& =\frac{1}{\alpha-\theta+1+\frac{\varepsilon}{q}}+\frac{1}{\beta-\theta+1+\frac{\varepsilon}{q}}-\frac{\varepsilon}{\left(\beta-\theta+1+\frac{\varepsilon}{q}\right)^{2}} \\
& =\frac{1}{\alpha-\theta+1}+\frac{1}{\beta-\theta+1}-o_{1}(1) .
\end{aligned}
$$

In addition, when $\gamma>0, \alpha, \beta>\theta-1$, notice the fact $e^{-\gamma t}<1, t \in[x, \infty)(x \geq 1)$. Making use of (10) and (11), we also obtain

$$
\begin{aligned}
\tilde{h} \cdot \varepsilon= & \varepsilon \int_{0}^{\infty} \int_{0}^{\infty} \frac{(\min \{1, x y\})^{\alpha}(\max \{1, x y\})^{\beta} \tilde{f}(x) \tilde{g}(y)}{e^{\gamma x y}} d x d y \\
= & \varepsilon \int_{1}^{\infty} x^{-1-\varepsilon} d x \int_{0}^{x} \frac{(\min \{1, t\})^{\alpha}(\max \{1, t\})^{\beta}}{e^{\gamma t}} t^{-\theta+\frac{\varepsilon}{q}} d t \\
= & \varepsilon \int_{1}^{\infty} x^{-1-\varepsilon} d x\left[\int_{0}^{1} e^{-\gamma t} t^{\alpha-\theta+\frac{\varepsilon}{q}} d t+\int_{1}^{x} e^{-\gamma t} t^{\beta-\theta+\frac{\varepsilon}{q}} d t\right] \\
= & \varepsilon \int_{1}^{\infty} x^{-1-\varepsilon} d x\left[\int_{0}^{1} e^{-\gamma t} t^{\alpha-\theta+\frac{\varepsilon}{q}} d t+\int_{1}^{\infty} e^{-\gamma t} t^{\beta-\theta+\frac{\varepsilon}{q}} d t-\int_{x}^{\infty} e^{-\gamma t} t^{\beta-\theta+\frac{\varepsilon}{q}} d t\right] \\
= & \frac{\gamma^{\frac{\theta-\alpha}{2}-1-\frac{\varepsilon}{2 q}} e^{-\frac{\gamma}{2}}}{1+\alpha-\theta+\frac{\varepsilon}{q}} M\left(\frac{\alpha-\theta}{2}+\frac{\varepsilon}{2 q}, \frac{\alpha-\theta}{2}+\frac{1}{2}+\frac{\varepsilon}{2 q}, \gamma\right) \\
& +\gamma^{\theta-\beta-1-\frac{\varepsilon}{q}} \Gamma\left(1+\beta-\theta+\frac{\varepsilon}{q}, \gamma\right) \\
& -\varepsilon \int_{1}^{\infty} x^{-1-\varepsilon} d x \int_{x}^{\infty} e^{-\gamma t} t^{\beta-\theta+\frac{\varepsilon}{q}} d t \\
> & \frac{\gamma^{\frac{\theta-\alpha}{2}-1-\frac{\varepsilon}{2 q}} e^{-\frac{\gamma}{2}}}{1+\alpha-\theta+\frac{\varepsilon}{q}} M\left(\frac{\alpha-\theta}{2}+\frac{\varepsilon}{2 q}, \frac{\alpha-\theta}{2}+\frac{1}{2}+\frac{\varepsilon}{2 q}, \gamma\right) \\
& +\gamma^{\theta-\beta-1-\frac{\varepsilon}{q}} \Gamma\left(1+\beta-\theta+\frac{\varepsilon}{q}, \gamma\right) \\
& -\varepsilon \int_{1}^{\infty} x^{-1} d x \int_{x}^{\infty} t^{\beta-\theta+\frac{\varepsilon}{q}} d t \\
= & {\left[\frac{\gamma^{\frac{\theta-\alpha}{2}-1} e^{-\frac{\gamma}{2}}}{1+\alpha-\theta} M\left(\frac{\alpha-\theta}{2}, \frac{\alpha-\theta}{2}+\frac{1}{2}, \gamma\right)+\gamma^{\theta-\beta-1} \Gamma(1+\beta-\theta, \gamma)\right]\left(1-o_{2}(1)\right) . }
\end{aligned}
$$

To sum up, we have

$$
\begin{aligned}
\tilde{h} \cdot \varepsilon & =\varepsilon \int_{0}^{\infty} \int_{0}^{\infty} \frac{(\min \{1, x y\})^{\alpha}(\max \{1, x y\})^{\beta} \tilde{f}(x) \tilde{g}(y)}{e^{\gamma x y}} d x d y \\
> & C(\alpha, \beta, \gamma, \theta)(1-o(1)) \quad\left(\varepsilon \rightarrow 0^{+}\right) .
\end{aligned}
$$

\section{Main results}

Theorem 1 If $p>1, \frac{1}{p}+\frac{1}{q}=1, \theta \in \mathbb{R}, f(x), g(y) \geq 0$, satisfying $0<\int_{0}^{\infty} x^{p \theta-1} f^{p}(x) d x<\infty, 0<$ $\int_{0}^{\infty} y^{q \theta-1} g^{q}(y) d y<\infty . \gamma \geq 0$, and parameters $\alpha, \beta, \gamma, \theta$ meet the following requirements: when $\gamma=0, \alpha>\theta, \beta<\theta-1$, and when $\gamma>0, \alpha, \beta>\theta-1$. Then the following inequality 
holds:

$$
\begin{aligned}
& \int_{0}^{\infty} \int_{0}^{\infty} \frac{(\min \{1, x y\})^{\alpha}(\max \{1, x y\})^{\beta} f(x) g(y)}{e^{\gamma x y}} d x d y \\
& \quad<C(\alpha, \beta, \gamma, \theta)\left\{\int_{0}^{\infty} x^{p \theta-1} f^{p}(x) d x\right\}^{\frac{1}{p}}\left\{\int_{0}^{\infty} y^{q \theta-1} g^{q}(y) d y\right\}^{\frac{1}{q}} .
\end{aligned}
$$

The constant $C(\alpha, \beta, \gamma, \theta)$ appearing on its right-hand side is the best possible, where $C(\alpha, \beta, \gamma, \theta)$ has the same expression as (12).

Proof By weighted Hölder's inequality (see [19]) and Lemma 1, we have

$$
\begin{aligned}
\int_{0}^{\infty} & \int_{0}^{\infty} \frac{(\min \{1, x y\})^{\alpha}(\max \{1, x y\})^{\beta} f(x) g(y)}{e^{\gamma x y}} d x d y \\
= & \int_{0}^{\infty} \int_{0}^{\infty} \frac{(\min \{1, x y\})^{\alpha}(\max \{1, x y\})^{\beta} f(x) g(y)}{e^{\gamma x y}}\left[\frac{y^{-\frac{\theta}{p}}}{x^{-\frac{\theta}{q}}}\right]\left[\frac{x^{-\frac{\theta}{q}}}{y^{-\frac{\theta}{p}}}\right] d x d y \\
\leq & \left\{\int_{0}^{\infty} \int_{0}^{\infty} \frac{(\min \{1, x y\})^{\alpha}(\max \{1, x y\})^{\beta} f^{p}(x)}{e^{\gamma x y}} \frac{y^{-\theta}}{x^{-\frac{p \theta}{q}}} d x d y\right\}^{\frac{1}{p}} \\
& \times\left\{\int_{0}^{\infty} \int_{0}^{\infty} \frac{(\min \{1, x y\})^{\alpha}(\max \{1, x y\})^{\beta} g^{q}(y)}{e^{\gamma x y}} \frac{x^{-\theta}}{y^{-\frac{q \theta}{p}}} d x d y\right\}^{\frac{1}{q}} \\
= & \left\{\int_{0}^{\infty} \omega(\alpha, \beta, \gamma, \theta, x) x^{p \theta-1} f^{p}(x) d x\right\}^{\frac{1}{p}}\left\{\int_{0}^{\infty} \omega(\alpha, \beta, \gamma, \theta, y) y^{q \theta-1} g^{q}(y) d y\right\}^{\frac{1}{q}} \\
= & C(\alpha, \beta, \gamma, \theta)\left\{\int_{0}^{\infty} x^{p \theta-1} f^{p}(x) d x\right\}^{\frac{1}{p}}\left\{\int_{0}^{\infty} y^{q \theta-1} g^{q}(y) d y\right\}^{\frac{1}{q}} .
\end{aligned}
$$

Now, suppose that " $\leq$ " in (16) takes the form of equality, then by the conclusion of Hölder's inequality, there exist constants $A$ and $B$, which are not all zero, such that

$$
A \frac{y^{-\theta}}{x^{-\frac{p \theta}{q}}} f^{p}(x)=B \frac{x^{-\theta}}{y^{-\frac{q \theta}{p}}} g^{q}(y) \quad \text { a.e. in }(0, \infty) \times(0, \infty)
$$

so there is a constant $C \neq 0$, the expression

$$
A x^{p \theta} f^{p}(x)=B y^{q \theta} g^{q}(y)=C \quad \text { a.e. in }(0, \infty) \times(0, \infty)
$$

is valid. Assuming that $A \neq 0$, we have $x^{p \theta-1} f^{p}(x)=\frac{C}{A x}$ a.e. in $(0, \infty)$. The integral as $\int_{0}^{\infty} \frac{C}{A x} d x$ is divergent, which contradicts the fact that $0<\int_{0}^{\infty} x^{p \theta-1} f^{p}(x) d x<\infty$. Hence expression (16) only takes the form of strict inequality.

We will prove by counter-proof that the constant factor $C(\alpha, \beta, \gamma, \theta)$ in (15) is the best possible. If the constant factor $C(\alpha, \beta, \gamma, \theta)$ in (15) is not the best possible, then there exists a positive number $K<C(\alpha, \beta, \gamma, \theta)$ such that inequality (15) is still valid when replacing $C(\alpha, \beta, \gamma, \theta)$ by $K$. But employing expressions (13) and (14), we get $C(\alpha, \beta, \gamma, \theta)(1-$ $o(1))<K$. Letting $\varepsilon \rightarrow 0^{+}$, it follows that $K \geq C(\alpha, \beta, \gamma, \theta)$, which contradicts the previous hypothesis that $K<C(\alpha, \beta, \gamma, \theta)$, so the constant factor $C(\alpha, \beta, \gamma, \theta)$ in (15) is the best possible. 
Theorem 2 Under the same conditions as Theorem 1, the inequality

$$
\begin{aligned}
& \int_{0}^{\infty} y^{\frac{p}{q}(1-q \theta)}\left\{\int_{0}^{\infty} \frac{(\min \{1, x y\})^{\alpha}(\max \{1, x y\})^{\beta} f(x)}{e^{\gamma x y}} d x\right\}^{p} d y \\
& <C^{p}(\alpha, \beta, \gamma, \theta) \int_{0}^{\infty} x^{p \theta-1} f^{p}(x) d x
\end{aligned}
$$

holds and the constant factor $C^{p}(\alpha, \beta, \gamma, \theta)$ appearing on its right-hand side is the best possible. In addition, inequality (17) is equivalent to inequality (15).

Proof First, we will derive (17) from (15).

Define a real function as $[f(x)]_{n}:=\min \{n, f(x)\}$. For $0<\int_{0}^{\infty} x^{p \theta-1} f^{p}(x) d x<\infty$, there exists $n_{0} \in \mathbb{N}$ such that $0<\int_{\frac{1}{n}}^{n} x^{p \theta-1} f^{p}(x) d x<\infty\left(n \geq n_{0}\right)$. Setting a real function as

$$
g_{n}(y):=y^{\frac{p}{q}(1-q \theta)}\left[\int_{\frac{1}{n}}^{n} \frac{(\min \{1, x y\})^{\alpha}(\max \{1, x y\})^{\beta}[f(x)]_{n}}{e^{\gamma x y}} d x\right]^{\frac{p}{q}} \quad\left(\frac{1}{n}<y<n, n \geq n_{0}\right)
$$

when $n \geq n_{0}$, making use of (15), we find

$$
\begin{aligned}
0 & <\int_{\frac{1}{n}}^{n} y^{q \theta-1} g_{n}^{q}(y) d y \\
& =\int_{\frac{1}{n}}^{n} y^{q \theta-1} g_{n}^{q-1}(y) g_{n}(y) d y \\
& =\int_{\frac{1}{n}}^{n} \int_{\frac{1}{n}}^{n} \frac{(\min \{1, x y\})^{\alpha}(\max \{1, x y\})^{\beta}[f(x)]_{n} g_{n}(y)}{e^{\gamma x y}} d x d y \\
& <C(\alpha, \beta, \gamma, \theta)\left\{\int_{\frac{1}{n}}^{n} x^{p \theta-1}[f(x)]_{n}^{p} d x\right\}^{\frac{1}{p}}\left\{\int_{\frac{1}{n}}^{n} y^{q \theta-1} g_{n}^{q}(y) d y\right\}^{\frac{1}{q}} .
\end{aligned}
$$

Moreover, making use of (18), we find

$$
\begin{aligned}
0 & <\int_{\frac{1}{n}}^{n} y^{q \theta-1} g_{n}^{q}(y) d y=\int_{\frac{1}{n}}^{n} y^{\frac{p}{q}(1-q \theta)}\left\{\int_{\frac{1}{n}}^{n} \frac{(\min \{1, x y\})^{\alpha}(\max \{1, x y\})^{\beta}[f(x)]_{n}}{e^{\gamma x y}} d x\right\}^{p} d y \\
& <C^{p}(\alpha, \beta, \gamma, \theta) \int_{\frac{1}{n}}^{n} x^{p \theta-1}[f(x)]_{n}^{p} d x<\infty .
\end{aligned}
$$

For $n \rightarrow \infty$, it follows that $0<\int_{0}^{\infty} y^{q \theta-1} g_{\infty}^{q}(y) d y<\infty$ and $0<\int_{0}^{\infty} x^{p \theta-1} f^{p}(x) d x<\infty$. By (15), we know that expressions (18) and (19) still keep the form of strict inequalities. Hence, inequality (17) holds.

Next, we will derive (15) from (17). If inequality (17) holds, by Hölder's inequality, we have

$$
\begin{aligned}
& \int_{0}^{\infty} \int_{0}^{\infty} \frac{(\min \{1, x y\})^{\alpha}(\max \{1, x y\})^{\beta} f(x) g(y)}{e^{\gamma x y}} d x d y \\
& \quad=\int_{0}^{\infty}\left[y^{\frac{1-q \theta}{q}} \int_{0}^{\infty} \frac{(\min \{1, x y\})^{\alpha}(\max \{1, x y\})^{\beta} f(x)}{e^{\gamma x y}} d x\right]\left[y^{\frac{q \theta-1}{q}} g(y)\right] d y \\
& \quad \leq\left\{\int_{0}^{\infty} y^{\frac{p(1-q \theta)}{q}}\left[\int_{0}^{\infty} \frac{(\min \{1, x y\})^{\alpha}(\max \{1, x y\})^{\beta} f(x)}{e^{\gamma x y}} d x\right]^{p} d y\right\}^{\frac{1}{p}}
\end{aligned}
$$




$$
\begin{aligned}
& \times\left\{\int_{0}^{\infty} y^{q \theta-1} g^{q}(y) d y\right\}^{\frac{1}{q}} \\
< & C(\alpha, \beta, \gamma, \theta)\left\{\int_{0}^{\infty} x^{p \theta-1} f^{p}(x) d x\right\}^{\frac{1}{p}}\left\{\int_{0}^{\infty} y^{q \theta-1} g^{q}(y) d y\right\}^{\frac{1}{q}} .
\end{aligned}
$$

It is shown above that inequality (15) holds, so inequality (17) is equivalent to inequality (15).

In addition, suppose that the constant factor $C^{p}(\alpha, \beta, \gamma, \theta)$ is not the best possible. Then by (17), the constant factor $C(\alpha, \beta, \gamma, \theta)$ we acquired in (15) is not the best possible too, which contradicts the conclusion of Theorem 1 . Thus the constant factor $C^{p}(\alpha, \beta, \gamma, \theta)$ in (17) is the best possible.

\section{Operator expression with norm}

Suppose that $p>1, \frac{1}{p}+\frac{1}{q}=1, \gamma \geq 0, \theta \in \mathbb{R}, f(x), g(y) \geq 0$, when $\gamma=0, \alpha>\theta, \beta<\theta-1$, and when $\gamma>0, \alpha, \beta>\theta-1$. $\varphi(x)=x^{p \theta-1}, \psi(y)=y^{q \theta-1}(x, y>0)$, apparently, $\psi^{1-p}(y)=y^{\frac{p}{q}(1-q \theta)}$. Now, define normed linear spaces as

$$
\begin{aligned}
& L_{\varphi}^{p}(0, \infty):=\left\{f:\|f\|_{p, \varphi}=\left[\int_{0}^{\infty} \varphi(x)|f(x)|^{p} d x\right]^{\frac{1}{p}}<\infty\right\}, \\
& L_{\psi}^{q}(0, \infty):=\left\{g:\|g\|_{q, \psi}=\left[\int_{0}^{\infty} \psi(y)|g(y)|^{q} d y\right]^{\frac{1}{q}}<\infty\right\}, \\
& L_{\psi^{1-p}}^{p}(0, \infty):=\left\{h:\|h\|_{p, \psi 1-p}=\left[\int_{0}^{\infty} \psi^{1-p}(y)|h(y)|^{p} d y\right]^{\frac{1}{p}}<\infty\right\} .
\end{aligned}
$$

If $f \in L_{\varphi}^{p}(0, \infty)$, a singular integral operator is defined as $T: L_{\varphi}^{p}(0, \infty) \rightarrow L_{\psi^{1-p}}^{p}(0, \infty)$,

$$
T(f)(y):=\int_{0}^{\infty} \frac{(\min \{1, x y\})^{\alpha}(\max \{1, x y\})^{\beta}}{e^{\gamma x y}} f(x) d x, \quad y \in(0, \infty)
$$

For $f \in L_{\varphi}^{p}(0, \infty), g \in L_{\psi}^{q}(0, \infty)$, the formal inner product of $T f$ and $g$ is defined as

$$
(T f, g):=\int_{0}^{\infty} \int_{0}^{\infty} \frac{(\min \{1, x y\})^{\alpha}(\max \{1, x y\})^{\beta} f(x) g(y)}{e^{\gamma x y}} d x d y
$$

With regard to (17), we have

$$
\|T(f)\|_{p, \psi^{1-p}}^{p}=\int_{0}^{\infty} \psi^{1-p}(y)|T(f)|^{p} d y<C^{p}(\alpha, \beta, \gamma, \theta)\|f\|_{p, \varphi}^{p}<\infty .
$$

According to the expression (20), the operator $T$ is bounded, that is,

$$
\|T\|:=\sup _{f(\neq 0) \in L_{\varphi^{1-p}}^{p}(0, \infty)} \frac{\|T(f)\|_{p, \varphi^{1-p}}}{\|f\|_{p, \varphi}} \leq C(\alpha, \beta, \gamma, \theta) .
$$

Because the constant factor $C(\alpha, \beta, \gamma, \theta)$ is optimal, therefore $\|T\|=C(\alpha, \beta, \gamma, \theta)$. 
Theorem 3 By the preceding Theorems 1 and 2, inequalities (15) and (17) can be expressed as the following operator expressions with norm:

$$
\begin{aligned}
& (T f, g)<\|T\|\|f\|_{p, \varphi}\|g\|_{q, \psi}, \\
& \|T(f)\|_{p, \psi^{1-p}}^{p}<\|T\|^{p}\|f\|_{p, \varphi}^{p} .
\end{aligned}
$$

\section{Simple applications}

We select the appropriate parameter values in (15) and (17) (first selecting the values of $\gamma$ and $\theta$, then determining the range of $\alpha$ and $\beta$ ) and calculate the value of constant factor $C(\alpha, \beta, \gamma, \theta)$ using Maple mathematical software. At the same time, combining with the representation methods of (21) and (22), some results in the references and some handsome Hilbert-type integral inequalities and their equivalent forms with single kernels or mixed kernels are obtained.

Example 1 Letting $\gamma=1, \theta=\frac{1}{2}, \alpha=\beta=0, p=q=2$, we can get $C\left(0,0,1, \frac{1}{2}\right)=\sqrt{\pi}$ by calculating formula (12). If $f, g>0, \varphi(x)=1$, satisfying $0<\|f\|_{2},\|g\|_{2}<\infty$, then we obtain (2) and its equivalent form

$$
\int_{0}^{\infty}\left[\int_{0}^{\infty} e^{-x y} f(x) d x\right]^{2} d y<\pi\|f\|_{2}^{2}
$$

where the constant factor $\pi$ is the best possible.

Example 2 Letting $\gamma=0, \theta=\frac{1}{2}, \alpha=1, \beta=-1, p=q=2$, we can get $C\left(1,-1,0, \frac{1}{2}\right)=\frac{8}{3}$ by calculating formula (12). If $f, g>0, \varphi(x)=1$, satisfying $0<\|f\|_{2},\|g\|_{2}<\infty$, then we have the equivalent inequalities

$$
\begin{aligned}
& \int_{0}^{\infty} \int_{0}^{\infty} \frac{\min \{1, x y\}}{\max \{1, x y\}} f(x) g(y) d x d y<\frac{8}{3}\|f\|_{2}\|g\|_{2}, \\
& \int_{0}^{\infty}\left[\int_{0}^{\infty} \frac{\min \{1, x y\}}{\max \{1, x y\}} f(x) d x\right]^{2} d y<\frac{64}{9}\|f\|_{2}^{2},
\end{aligned}
$$

where the constant factors $\frac{8}{3}, \frac{64}{9}$ are the best possible.

Example 3 Letting $\gamma=0, \theta=-\frac{1}{2}, \alpha=0, \beta=-2, p=q=2$, we get $C\left(0,-2,0,-\frac{1}{2}\right)=\frac{8}{3}$ by calculating formula (12). If $f, g>0, \varphi(x)=\frac{1}{x^{2}}$, satisfying $0<\|f\|_{2, \varphi},\|g\|_{2, \varphi}<\infty$, then we have the equivalent inequalities

$$
\begin{aligned}
& \int_{0}^{\infty} \int_{0}^{\infty} \frac{f(x) g(y)}{(\max \{1, x y\})^{2}} d x d y<\frac{8}{3}\|f\|_{2, \varphi}\|g\|_{2, \varphi} \\
& \int_{0}^{\infty} y^{2}\left[\int_{0}^{\infty} \frac{f(x)}{(\max \{1, x y\})^{2}} d x\right]^{2} d y<\frac{64}{9}\|f\|_{2, \varphi}^{2}
\end{aligned}
$$

where the constant factors $\frac{8}{3}, \frac{64}{9}$ are the best possible.

Example 4 Letting $\gamma=0, \theta=\frac{3}{2}, \alpha=2, \beta=0, p=q=2$, we get $C\left(2,0,0, \frac{3}{2}\right)=\frac{8}{3}$ by calculating formula (12). If $f, g>0, \varphi(x)=x^{2}$, satisfying $0<\|f\|_{2, \varphi},\|g\|_{2, \varphi}<\infty$, then we have the 
equivalent inequalities

$$
\begin{aligned}
& \int_{0}^{\infty} \int_{0}^{\infty}(\min \{1, x y\})^{2} f(x) g(y) d x d y<\frac{8}{3}\|f\|_{2, \varphi}\|g\|_{2, \varphi}, \\
& \int_{0}^{\infty} \frac{1}{y^{2}}\left[\int_{0}^{\infty}(\min \{1, x y\})^{2} f(x) d x\right]^{2} d y<\frac{64}{9}\|f\|_{2, \varphi}^{2}
\end{aligned}
$$

where the constant factors $\frac{8}{3}, \frac{64}{9}$ are the best possible.

Example 5 Letting $\gamma=1, \theta=0, \alpha=1, \beta=0, p=q=2$, we get $C(1,0,1,0)=\frac{e-1}{e}$ by calculating formula (12). If $f, g>0, \varphi(x)=\frac{1}{x}$, satisfying $0<\|f\|_{2, \varphi},\|g\|_{2, \varphi}<\infty$, then we have the equivalent inequalities

$$
\begin{aligned}
& \int_{0}^{\infty} \int_{0}^{\infty} \frac{\min \{1, x y\} f(x) g(y)}{e^{x y}} d x d y<\frac{e-1}{e}\|f\|_{2, \varphi}\|g\|_{2, \varphi} \\
& \int_{0}^{\infty} y\left[\int_{0}^{\infty} \frac{\min \{1, x y\} f(x)}{e^{x y}} d x\right]^{2} d y<\left(\frac{e-1}{e}\right)^{2}\|f\|_{2, \varphi}^{2}
\end{aligned}
$$

where the constant factors $\frac{e-1}{e},\left(\frac{e-1}{e}\right)^{2}$ are the best possible.

Example 6 Letting $\gamma=1, \theta=0, \alpha=0, \beta=1, p=q=2$, we get $C(0,1,1,0)=\frac{e+1}{e}$ by calculating formula (12). If $f, g>0, \varphi(x)=\frac{1}{x}$, satisfying $0<\|f\|_{2, \varphi},\|g\|_{2, \varphi}<\infty$, then we have the equivalent inequalities

$$
\begin{aligned}
& \int_{0}^{\infty} \int_{0}^{\infty} \frac{\max \{1, x y\} f(x) g(y)}{e^{x y}} d x d y<\frac{e+1}{e}\|f\|_{2, \varphi}\|g\|_{2, \varphi}, \\
& \int_{0}^{\infty} y\left[\int_{0}^{\infty} \frac{\max \{1, x y\} f(x)}{e^{x y}} d x\right]^{2} d y<\left(\frac{e+1}{e}\right)^{2}\|f\|_{2, \varphi}^{2}
\end{aligned}
$$

where the constant factors $\frac{e+1}{e},\left(\frac{e+1}{e}\right)^{2}$ are the best possible.

Example 7 Letting $\gamma=1, \theta=\frac{1}{2}, \alpha=1, \beta=-1, p=q=2$, we get $C\left(1,-1,1, \frac{1}{2}\right)=$ $\frac{\sqrt{\pi}}{2}(5 \operatorname{erf}(1)-4)+e^{-1}=0.5570924045^{+}$by calculating formula (12). If $f, g>0, \varphi(x)=1$, satisfying $0<\|f\|_{2, \varphi},\|g\|_{2, \varphi}<\infty$, then we have the equivalent inequalities

$$
\begin{aligned}
& \int_{0}^{\infty} \int_{0}^{\infty} \frac{\min \{1, x y\} f(x) g(y)}{\max \{1, x y\} e^{x y}} d x d y<\left(\frac{\sqrt{\pi}}{2}(5 \operatorname{erf}(1)-4)+e^{-1}\right)\|f\|_{2, \varphi}\|g\|_{2, \varphi}, \\
& \int_{0}^{\infty}\left[\int_{0}^{\infty} \frac{\min \{1, x y\} f(x)}{\max \{1, x y\} e^{x y}} d x\right]^{2} d y<\left(\frac{\sqrt{\pi}}{2}(5 \operatorname{erf}(1)-4)+e^{-1}\right)^{2}\|f\|_{2, \varphi}^{2},
\end{aligned}
$$

where the constant factors $\frac{\sqrt{\pi}}{2}(5 \operatorname{erf}(1)-4)+e^{-1},\left(\frac{\sqrt{\pi}}{2}(5 \operatorname{erf}(1)-4)+e^{-1}\right)^{2}$ are the best possible, and $\operatorname{erf}(x)=\frac{2}{\sqrt{\pi}} \int_{0}^{x} e^{-t^{2}} d t$ is an error function.

\section{Acknowledgements}

The author is extremely grateful to the reviewers for critical reading of the manuscript and making valuable comments and suggestions leading to an overall improvement of the paper.

\section{Funding}


Competing interests

The author declares that they have no competing interests.

\section{Authors' contributions}

The author read and approved the final version of the manuscript.

\section{Publisher's Note}

Springer Nature remains neutral with regard to jurisdictional claims in published maps and institutional affiliations.

Received: 21 November 2018 Accepted: 21 March 2019 Published online: 02 April 2019

\section{References}

1. Hardy, G.H., Littlewood, J.E., Pólya, G.: Inequalities. Cambridge University Press, Cambridge (1952)

2. Mitrinović, D.S., Pečarić, J.E., Fink, A.M.: Inequalities Involving Functions and Their Integrals and Derivatives. Kluwer Academic, Boston (1991)

3. Yang, B.C.: The Norm of Operator and Hilbert-Type Inequalities. Science Press, Beijing (2009) (in Chinese)

4. Rassias, M.T., Yang, B.C.: On a multidimensional Hilbert-type integral inequality associated to the gamma function. Appl. Math. Comput. 249, 408-418 (2014)

5. Yang, B.C., He, B.: A new half-discrete Hilbert-type inequality in the whole plane. J. Appl. Anal. Comput. 7(3), 977-991 (2017)

6. Liu, Q., Sun, W.B.: A Hilbert-type integral inequality with the mixed kernel of multi-parameters. C. R. Acad. Sci. 351, 605-611 (2013)

7. Liu, Q., Chen, D.Z.: A Hilbert-type integral inequality with a hybrid kernel and its applications. Colloq. Math. 143(2), 193-207 (2016)

8. Rassias, M.T., Yang, B.C.: On a Hardy-Hilbert-type inequality with a general homogeneous kernel. Int. J. Nonlinear Anal. Appl. 7(1), 249-269 (2016)

9. Liu, Q.: A Hilbert-type fractal integral inequality and its applications. J. Inequal. Appl. 2017, 83 (2017)

10. Liu, Q.: A Hilbert-type fractional integral inequality with the kernel of Mittag-Leffler function and its applications. Math. Inequal. Appl. 21(3), 729-737 (2018)

11. Hong, R., Wen, Y.M.: Necessary and sufficient condition for a class of Hilbert-type integral inequality with a non-homogeneous kernel and its application. J. Jilin Univ. Sci. Ed. 56(2), 227-232 (2018) (in Chinese)

12. Rassias, M.T., Yang, B.C.: On a multidimensional half-discrete Hilbert-type inequality related to the hyperbolic cotangent function. Appl. Math. Comput. 242, 800-813 (2014)

13. Chen, Q., Yang, B.C.: A survey on the study of Hilbert-type inequalities. J. Inequal. Appl. 2015, 302 (2015)

14. Yang, B.C., Debnath, J.: Half-Discrete Hilbert-Type Inequalities. World Scientific, Singapore (2014)

15. Yang, B.C.: Hilbert-Type Integral Inequalities. Bentham Science Publishers Ltd (2010)

16. Yang, B.C.: Hilbert-type integral inequality with non-homogeneous kernel. J. Shanghai Univ. Nat. Sci. 17(5), 603-605 (2011) (in Chinese)

17. Huang, Z.S., Guo, D.R.: An Introduction to Special Function. Beijing Press, Beijing (2000) (in Chinese)

18. Kuang, J.C.: Real Analysis and Functional Analysis. Higher Education Press, Beijing (2014) (in Chinese)

19. Kuang, J.C.: Applied Inequalities. Shangdong Science Technic Press, Jinan (2004) (in Chinese)

\section{Submit your manuscript to a SpringerOpen ${ }^{\odot}$ journal and benefit from:}

- Convenient online submission

- Rigorous peer review

- Open access: articles freely available online

- High visibility within the field

- Retaining the copyright to your article

Submit your next manuscript at $\boldsymbol{~ s p r i n g e r o p e n . c o m ~}$ 